\title{
Préserver le milieu naturel par la maîtrise des déversements
}

\author{
par Hugues Hartleyb \\ Président du S.I.V.O.M. de l'Ehn
}

Alain Onnee

Ingénieur à BEREST S.A.

\section{INTRODUCTION}

\begin{abstract}
A quelques dizaines de kilomètres au sud-ouest de Strasbourg, six communes de l'amont de la vallée de l'Ehn dont Obernai, la plus importante, se sont progressivement équipées jusque vers 1970, d'un assainissement général unitaire gravitaire. Les collecteurs intercommunaux, construits par la suite, relient d'une façon simple l'aval du réseau unitaire de la commune d'amont à l'amont du réseau unitaire de la commune d'aval, eu égard aux contraintes technique et économique. Ils assurent le transport des eaux résiduaires de temps sec et d'une partie des eaux de ruissellement pluviales jusqu'à la station d'épuration. Cette dernière, du type à boues activées en aération prolongée, construite en bordure de l'Ehn à l'aval de Niedemai, traite depuis 1979 une pollution urbaine et industrielle voisine de 20000 éqhab.
\end{abstract}

\section{LE PROBLÈME POSÉ ET PREMIÈRE ÉTUDE}

En raison des mises en charge répétées du collecteur de liaison d'Obernai à la station d'épuration qui produisirent en 1981-82 des inondations de sous-sols aménagés d'immeubles riverains dans la traversée du village de Niedernai, le S.I.V.O.M. entreprit une étude du réseau intercommunal dans laquelle était évoquée pour la première fois la construction d'un bassin de rétention de pollution à l'aval d'Obernai.

En Alsace à cette époque, la protection de la qualité des eaux de la rivière était appréhendée en corollaire à l'hydraulique des réseaux, selon les prescriptions de la règle A.T.V. 128 de juillet 1977, applicable en Allemagne et adoptée comme guide de référence.

La restructuration essentielle était conceptuelle et concernait le principe de raccordement des bassins de collecte:

- les collecteurs intercommunaux devaient être développés en un réseau continu, distinct des réseaux communaux. Ainsi, la qualité des effluents de transit ne serait plus modifiée par suppressions des phases de dilution et de décharge successives et la maîtrise plus précise du débit conservé,

- après redistribution, les bassins de collecte seraient raccordés au collecteur intercommunal au travers d'un déversoir d'orage latéral à crête haute unique, associé à un

L'amélioration de la qualité des eaux de l'Ehn, petit affluent de l'Ill qui arrose Obernai, passe par la mâtrise des déversements par temps de pluie du réseau d'assainissement unitaire. La modélisation informatique par KOSIM du transport de pollution en réseau et l'exploitation simple des résultats par la méthode de l'Agence de l'Eau Rhin-Meuse composent un outil d'aide à la décision intéressant, utilisé pour la première fois en Alsace. 
dispositif de limitation de débit et un bassin de rétention de pollution, calculés pour une pluie d'intensité $15 \mathrm{l} / \mathrm{s}$.ha,

- le débit de transit serait limité en fonction des capacités hydrauliques des collecteurs existants et de la station d'épuration et un bassin de rétention de pollution de $5210 \mathrm{~m}^{3}$ (solution 2) serait construit à l'aval d'Obernai.

Un programme de travaux s'ensuivit mais, la construction du bassin de pollution fut différée en raison de son coût. A la place, un déversoir d'orage fut construit pour régler temporairement le problème hydraulique.

\section{L'ÉTU L'TUE-DIAGNOSTIC ET LE PRO- GRAMME PLURIANNUEL DE TRA- VAUX}

Ce n'est que depuis les deux inventaires de 1976 et 1981 que sont appréciés réellement et d'une manière fiable la qualité des eaux superficielles et l'impact des réseaux d'assainissement des collectivités locales.

L'Ehn, affluent de la rive gauche de l'Ill, prend sa source dans les Vosges à $13 \mathrm{~km}$ à l'ouest d'Obernai. Il draine un bassin versant de $57,6 \mathrm{~km}^{2}$ occupé à plus de $50 \%$ par la forêt vosgienne. Cette rivière, qui réagit rapidement aux précipitations du fait de la nature granito-gréso-loessique et de l'hypsométrie marquée de son bassin versant, connaît des étiages prolongés faibles $\left(\mathrm{F} 1 / 2=0,2 \mathrm{~m}^{3} / \mathrm{s}\right.$ ) de mai à septembre. Elle constitue le milieu récepteur des eaux de ruissellement et des rejets des agglomérations riveraines. Un objectif de qualité 2 passable lui était assigné par arrêté préfectoral le 23 octobre 1985.

La qualité des eaux sur son cours aval s'est améliorée depuis que les jus, produits par l'activité choucroutière sont acheminés vers la nouvelle station d'épuration de Strasbourg.

Cependant, un déclassement d'un rang au moins subsiste encore en aval de la station d'épuration du S.I.V.O.M. à Niedernai.

Outre l'usage essentiel piscicole à préserver, traduit par une classification de la rivière en $2^{2}$ catégorie (eaux cyprinicoles), il s'agit également d'améliorer les aspects sanitaire et esthétique. En effet, le cours d'eau, qui traverse quelques agglomérations avant sa confluence avec l'Ill, participe à la qualité de ces sites (hygiène, aspect, odeurs, colonisations nuisibles animales et végétales, etc.).

Dès son cinquième programme d'activité (1987-1991), 1'Agence de l'Eau Rhin-Meuse a inscrit une somme importante à son budget pour l'aide financière aux étudesdiagnostic sur réseaux.

En mars 1991, le S.I.V.O.M. entreprenait, avec le concours financier de l'Agence de l'Eau Rhin-Meuse et le Conseil Général du Bas-Rhin, une étude diagnostic visant à faire le point de l'assainissement et du traitement des eaux existants, de l'état du milieu naturel et proposer un schéma directeur dont l'un des chapitres est consacré à l'amélioration du transport.

\section{DÉMARCHE SUIVIE}

L'étude-diagnostic, achevée à la fin du premier semestre 92, reprenait les principes de base de la restructuration proposée dans l'étude antérieure. Le transport de pollution fut abordé non plus sous l'angle hydraulique mais, ce qui était nouveau, sous l'aspect du flux déversé.

Ce travail a été réalisé au moyen du logiciel SPEIKAKOSIM, développé par l'Université de Hanovre, pour une série pluviométrique de longue durée.

\section{$\mathrm{V}$ DESCRIPTION SOMMAIRE DU MO- DÈLE}

KOSIM est un modèle global applicable aux réseaux ramifiés. La partie hydraulique utilise le modèle à plusieurs réservoirs linéaires en cascade et prend en compte les surfaces imperméables et perméables, les périodes sèches et pluvieuses. La partie transport de pollution repose sur la composition de trois sources indépendantes - eaux usées, eaux pluviales des surfaces perméables et eaux pluviales des surfaces imperméables - et sur l'hypothèse des concentrations constantes des eaux pluviales dans le temps.

Le paramètre étudié est la DCO avec des concentrations moyennes standard de $107 \mathrm{mg} / \mathrm{l}$ pour les eaux pluviales et $600 \mathrm{mg} / \mathrm{l}$ pour les eaux usées domestiques.

La sédimentation et l'effet de rinçage dans le réseau sont pris en compte par une loi simplifiée où la sédimentation est calculée en fonction du temps tandis que le rinçage dépend du débit instantané. Les algorithmes utilisés ne sont pas détaillés ici. On retient les hypothèses suivantes: aucun dépôt dans le réseau à l'état initial et quantité maximale sédimentable égale à $3,5 \mathrm{~kg}$ DCO par hectare imperméabilisé.

Le bassin de rétention de pollution d'Obernai est décrit comme un ouvrage en parallèle avec déversoir de traitement. La vitesse de sédimentation est constante et égale à $1 \mathrm{~m} / \mathrm{s}$ avec une efficacité maximale de $70 \%$.

\section{BASE DE DONNÉES PLUVIOMÉTRI- QUES}

La série de pluies utilisée correspond aux précipitations réelles observées au cours de la période 1968-1977 à la station météorologique de Strasbourg-Entzheim, distante de quinze kilomètres au nord-est, réduite aux cinq mois d'étiage des rivières vosgiennes, de mai à septembre : soit encore 153 jours et discrétisée au pas de temps de $5 \mathrm{mi}$ nutes. La hauteur moyenne annuelle précipitée sur la période est de $301 \mathrm{~mm}$ en 48 événements distincts.

\section{RÉSULTATS DE KOSIM}

On observe l'évolution de la concentration du rejet ainsi que l'efficacité du système d'assainissement en volume et en charge annuels, en faisant varier le volume utile du bassin de pollution. Ces résultats sont illustrés par la figure 1 . On remarque que l'efficacité tend rapidement vers une asymptote voisine de $80 \%$ sur la DCO. Pour dépasser cette valeur, il faudrait réduire le déversement par les déversoirs d'orage soit en renforçant de façon importante les sections des collecteurs de transport soit en mettant en place des capacités spécifiques de stockage au sein même 
du réseau, ce qui poserait de nombreux problèmes d'implantation, de gestion et d'exploitation. On est amené à choisir un optimum.

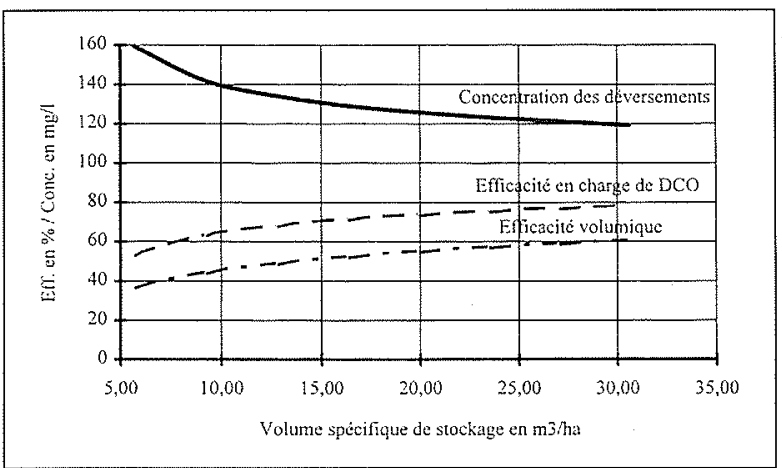

1. Courbe volume spécifique stocké - efficacité.

\section{APPLICATION DE LA MÉTHODE DE L'AGENCE DE L'EAU RHIN- MEUSE}

A l'aide d'un module additionnel, les déversements cumulés par événement, caractérisés par le volume déversé $\left(\mathrm{d} V_{i}\right)$, le flux polluant $\left(\mathrm{d} F_{i}\right)$ et la durée $\left(\mathrm{d} t_{i}\right)$, issus de KOSIM, sont additionnés au volume $\left(Q^{*} \mathrm{~d} t_{i}\right)$ et au flux propre ( $C^{*} Q^{*} \mathrm{~d} t_{i}$ ) écoulé par la rivière pendant le même temps. Dans le cas présent le débit $(Q)$ correspond au débit moyen mensuel d'étiage de fréquence 0,5 et la concentration $(C)$ à l'amont du rejet à la moyenne des valeurs des paramètres physico-chimiques de la classe de qualité $1 \mathrm{~B}$, objectif fixé à l'amont du rejet. La concentration moyenne $\left(C_{m}\right)$ à l'aval du rejet est obtenue par la conservation des masses:

$$
C_{m}=\left(\mathrm{d} F_{i}+C^{*} Q^{*} \mathrm{~d} t_{i}\right) /\left(\mathrm{d} V_{i}+Q^{*} \mathrm{~d} t_{i}\right)
$$

Pour estimer la durée d'impact $\left(\Sigma \mathrm{d} t_{e f}\right)$ du déversement sur la qualité de l'eau de la rivière, on adopte l'hypothèse simplificatrice qui consiste à ajouter à chaque déversement une durée empirique minimale d'effet, en l'absence d'observation réelle du pouvoir auto-épuratoire du cours d'eau. Cette durée, au regard de l'oxygène dissous et de l'azote, est de l'ordre de $12 \mathrm{~h}\left(\mathrm{~d} t_{t f}=\mathrm{d} t_{i}+12\right)$. Toutefois, si le temps qui sépare la fin d'un déversement et le début du suivant est inférieure à celle-ci, on retient la valeur réelle $\left(\mathrm{d} t_{c f} \leqslant \mathrm{~d} t_{i}+12\right)$.

Les durées d'impact sont alors sériées en trois catégories en fonction de la concentration moyenne $\left(C_{m}\right)$ de chaque événement par rapport à deux seuils $\left(C_{1}\right)$ et $\left(C_{2}\right)$ qui représentent respectivement la concentration limite supérieure du rang de la classe objectif de qualité (classe 2 : $\mathrm{DCO}=40 \mathrm{mgO}_{2} / 1$ ) et concentration moyenne du rang de qualité objectif inférieur (milieu classe 3: $\mathrm{DCO}=60 \mathrm{mgO}_{2} / 1$ ). Le total des durées des catégories correspondant à $C_{1}<C_{m} \leqslant C_{2}$ et $C_{m}>C_{2}$ est moyenné par le nombre d'années (10 ans) puis comparé à la durée d'étiage ( 153 jours) pour exprimer un taux de déclassement : respectivement $\tau_{1}$ et $\tau_{2}$. On doit vérifier simultanément les deux conditions suivantes: pour la classe $C_{1}<C_{m} \leqslant C_{2}$ :

$$
\tau_{1} \%=100 * \sum \mathrm{d} t_{e f} / 153 \text { avec } \tau_{1} \leqslant 5 \%
$$

pour la classe $C_{m}>C_{2}$ :

$$
\tau_{2} \%=100 * \sum \mathrm{d} t_{e f} / 153 \text { avec } \tau_{2} \leqslant 10 \%
$$

Les résultats des calculs effectués pour différents volumes de bassin et éventuellement des débits conservés par les déversoirs d'orage sont traduits par une courbe volumeefficacité d'aide à la décision (fig. 2).

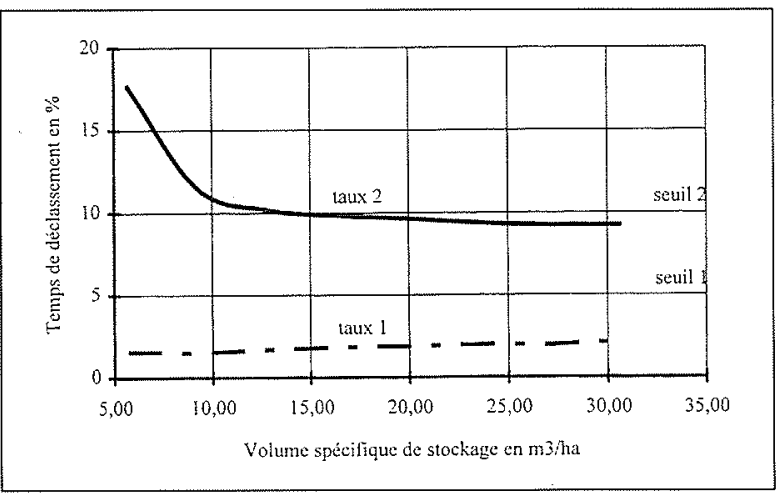

2. Courbe volume spécifique stocké - temps de déclassement.

Cette étude de sensibilité basée sur la conservation massique de la pollution montre que, pour atteindre l'objectif, la modélisation a conduit à retenir un volume utile de $4800 \mathrm{~m}^{3}$ correspondant à une capacité spécifique de $22,5 \mathrm{~m}^{3} / \mathrm{ha}_{\text {inip }}$ en un bassin unique pour le bassin de collecte d'Obernai.

\section{CONCEPTION DE L'OUVRAGE D'OBERNAI (fig. 3)}

L'ouvrage de type «parallèle » avec déversoir de traitement fonctionne gravitairement. Il comprend 2 files de deux bassins en série de $1200 \mathrm{~m}^{3}, 55 \mathrm{~m}$ de long, $8 \mathrm{~m}$ de large, 2,46 $\mathrm{m}$ de hauteur d'eau utile, alimentées en cascade par surverse, permettant le remplissage séquentiel. Le débit conservé à l'aval du bassin est limité à $1.40 \mathrm{l} / \mathrm{s}$ par un dispositif à ouverture variable à opercule et flotteur, modifiable à $260 \mathrm{l} / \mathrm{s}$ pour la situation future.

La géométrie rectangulaire simple du bassin permettra un fonctionnement en débourbeur sous une charge hydraulique inférieure à $5 \mathrm{~m}^{3} / \mathrm{m}^{2}$.h et une vitesse traversière horizontale inférieure à $6 \mathrm{~cm} / \mathrm{s}$ au débit de pointe future de $2,2 \mathrm{~m}^{3} / \mathrm{s}$. Actuellement, ces mêmes paramètres ne dépassent pas $2,5 \mathrm{~cm} / \mathrm{s}$ et $2 \mathrm{~m}^{3} / \mathrm{m}^{2}$.h au débit maximal de $1 \mathrm{~m}^{3} / \mathrm{s}$, limité par le collecteur d'amenée existant dont le renforcement est prévu ultérieurement.

Le nettoyage est réalisé automatiquement en fin de vidange du bassin à l'aide de quatre augets de rinçage basculants $8 \mathrm{~m}$ de longueur remplis chacun de $12 \mathrm{~m}^{3}$ d'eau 


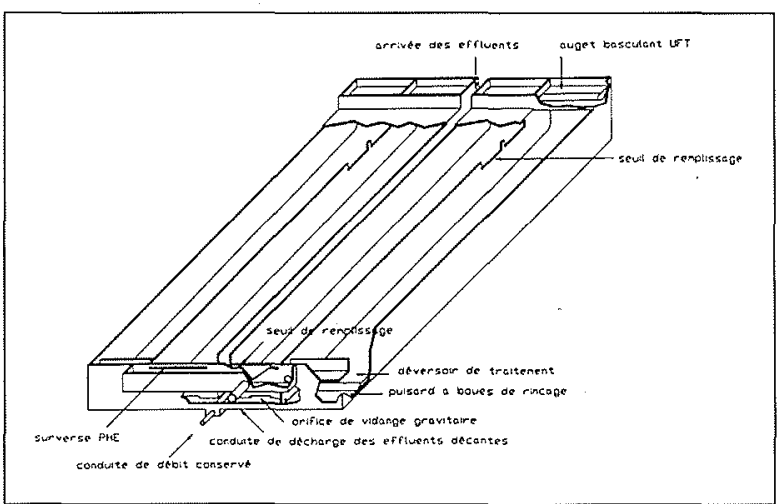

3. Isométrie schématique du bassin.

de la rivière par pompage automatique. Les boues décantées chassées sont évacuées avec les effluents vers la station d'épuration.

\section{$X$ LES ÉQUIPEMENTS D'AUTOCON- TRÔLE}

Le matériel d'autocontrôle installé permet de connâttre le fonctionnement en temps réel du bassin de pollution des points de vues hydraulique et pollution, soit sur site, soit à distance par minitel (fig. 4).

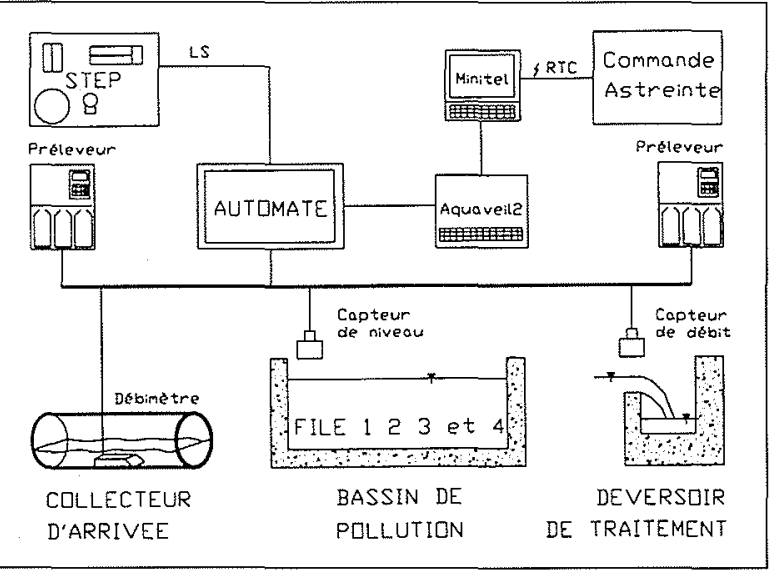

\section{L'autocontrôle.}

Un capteur combiné hauteur/vitesse, comprenant une sonde piézorésistive de mesure de hauteur et une sonde ultrasons de mesure de vitesse, placé sur le collecteur d'arrivée permet de connaître le débit affluent quel que soit le régime d'écoulement. Quatre ensembles de mesure de niveau à impulsions soniques indiquent à tout moment l'état de remplissage des compartiments du bassin. Le déversement est suivi par deux ensembles de mesures de débit à impulsions soniques placées au-dessus des déversoirs de traitement.

Un échantillonnage des effluents bruts et déversés est effectué par deux préleveurs automatiques thermostatés et réfrigérés.
L'ensemble de l'installation est gérée par automate programmable.

La connexion d'un minitel avec l'Aquaveil II de télégestion permet de visualiser les courbes des niveaux, des débits annsi que les états de marche des pompes d'eau claire.

\section{LA CONSTRUCTION}

La construction, inscrite dès la première année du contrat pluriannuel de travaux mis au point à l'issue de l'étude diagnostic engageant S.I.V.O.M. et ses deux partenaires financiers: l'Agence de l'Eau Rhin-Meuse et le Conseil Général du Bas-Rhin, s'est achevée à la fin du premier semestre 1994.

Le bassin couvert, semi-enterré et sa parcelle ont reçu un aménagement paysager végétal soigné afin de réduire les impacts négatifs et favoriser son intégration au site.

\section{I LE FINANCEMENT}

Le financement a été mis en place pour une réalisation en une tranche unique. Le coût total des travaux qui s'établit à $8500000 \mathrm{~F} \mathrm{H}$.T. se répartit en :

- travaux préparatoires:

- terrassement et génie-civil :

5750000

- équipement :

1100000

- matériel d'autocontrôle:

400000

- aménagement extérieur et paysager :

450000

- honoraires :

500000

Les trois cosignataires du contrat pluriannuel ont participé au financement dans les formes suivantes:

- l'Agence de l'Eau Rhin-Meuse sous forme de prêts bonifiés à hauteur de $3100000 \mathrm{~F}$, remboursables annuellement par dixième jusqu'à l'obtention de l'objectif fixé (en pratique la fin du contrat),

- le Conseil Général du Bas-Rhin sous forme de subventions à hauteur de $2600000 \mathrm{~F}$.

- le S.I.V.O.M. pour le solde.

\section{L'EXPLOITATION ET LE SUIVI}

Depuis sa mise en service, l'exploitation du bassin et son suivi sont assurés par l'agence locale de la Société Lyonnaise des Eaux qui exploite déjà la station d'épuration et les collecteurs intercommunaux.

Après un an, les travaux d'entretien sont limités à :

- un nettoyage hebdomadaire du dégrillage statique d'entrée,

- un nettoyage semestriel des parois,

- un étalonnage annuel des sondes,

- un contrôle hebdomadaire du fonctionnement en absence de pluie.

L'analyse des flux piégés et déversés porte sur la DCO et les MES par homogénéité avec les paramètres d'autocontrôle retenus à la station d'épuration. Il est procédé : 
- en période d'étiage de l'Ehn : de mai à septembre inclus, à la mesure des volumes et des flux DCO et MES sur échantillon moyen reconstitué, à chaque épisode pluvieux produisant le fonctionnement du déversoir de traitement ou non,

- d'octobre à avril inclus, aux mêmes mesures limitées aux épisodes avec déversement.

Les premiers résultats sont présentés par la suite dans l'article «Bilan du fonctionnement du bassin d'orage du S.I.V.O.M. de I'Ehn»».

\section{XIV 돔 CONCLUSION}

La dégradation de la qualité des eaux des rivières s'effectue de façon insidieuse dans l'ignorance et la responsabilité collective. Heureusement, depuis une dizaine d'années, de nombreux acteurs se sont mobilisés, certains d'abord pour éveiller l'attention, d'autres ensuite pour concevoir, réaliser et financer des équipements d'assainissement adaptés. Les travaux à engager, bien que s'étalant sur plusieurs années, concernent des programmes de financement longs et coûteux qui ont des répercussions souvent importantes sur le prix de leau. L'optimisation de la démarche au moyen d'outils et de méthodes de calcul modernes aide le décideur à choisir sur des critères plus réalistes.

Après une année d'exploitation, les résultats obtenus sont encourageants et montrent le piégeage d'importants flux polluants, bien que l'ouvrage ne soit pas utilisé à sa capacité nominale et que des travaux restent encore à entreprendre sur le réseau. L'observation du milieu naturel, effectuée dans le cadre des inventaires réguliers de la qualité des eaux des rivières, permettra de suivre l'amélioration physico-chimique de l'eau mais également sa qualité biologique ainsi que celle des sédiments. 